\title{
SOUNDSCAPE DESIGN IN CITY PARKS: EXPLORING THE RELATIONSHIPS BETWEEN SOUNDSCAPE COMPOSITION PARAMETERS AND PHYSICAL AND PSYCHOACOUSTIC PARAMETERS
}

\author{
Jiang LIU,a, b, Jian KANG \\ ${ }^{a}$ School of Architecture, Fuzhou University, Qi Shan Campus, 2 Xue Yuan Road, \\ University Town, Fuzhou, Fujian 350116 P. R. China \\ ${ }^{b}$ School of Architecture, University of Sheffield, Western Bank, Sheffield S10 2TN, UK
}

Submitted 21 May 2014; accepted 12 Dec. 2014

\begin{abstract}
Soundscape design in city parks is important for a better visiting experience. The aim of this research is to find a more effective way to design soundscapes in city parks, by exploring the relationships between certain physical and psychoacoustic parameters and soundscape composition parametersas proposed in this study, including perceived loudness of individual sound (PLS), perceived occurrences of individual sound (POS), and soundscape diversity index (SDI). The parameters were based on soundscape information gathered with a specifically designed soundwalk method in five city parks in Xiamen, China. The results showed that the soundscape composition parameters play important roles as soundscape parameters. $\mathrm{L}_{\mathrm{Ceq}}-\mathrm{L}_{\mathrm{Aeq}}, \mathrm{L}_{\mathrm{A} 10}-\mathrm{L}_{\mathrm{A} 90}$ and sharpness showed more explanatory power to the soundscape composition parameters of individual sound categories than other physical and psychoacoustic parameters. PLS of human sounds was the most frequently introduced variable for nearly all the other objective parameters, followed by SDI. Some of the soundscape composition parameters were found to be mutually explainable, including PLS of human sounds with $\mathrm{L}_{\mathrm{A} 10}, \mathrm{~L}_{\mathrm{Ceq}}-\mathrm{L}_{\mathrm{Aeq}}$ and sharpness, respectively, POS of traffic sounds with $\mathrm{L}_{\mathrm{A} 10}$, and PLS of both mechanical and geophysical sounds with sharpness, which supply important information for soundscape design in city parks.
\end{abstract}

Keywords: soundscape design, soundscape evaluation, soundwalk, soundscape composition parameter, city park.

\section{Introduction}

The soundscape concept has been drawing increasing attention in the field of landscape planning and design (Fowler 2013; Liu et al. 2014, 2013a). However, effective approaches for soundscape design are still limited, which could be largely attributed to the lack of suitable soundscape evaluation/characterisation parameters. Currently, standardised assessment methods of urban sounds/ soundscapes from the EU Directive on environmental noise are typically focusing on objective noise quantification defined through physical parameters such as $\mathrm{L}_{\text {Aeq }}$ (sound level with the same energy content as the varying acoustic signal measured, A-weighted) and $\mathrm{L}_{\text {den }}$ (Day-evening-night equivalent level, A-weighted). These parameters provide a constant filter that is independent of sound source identification, therefore the information reflected by them could be misleading for planners (Raimbault,
Dubois 2005). This is mainly because soundscapes are perceived by human and shaped by neural and psychological attributes rather than by physical parameters (Matsinos et al. 2008). Although it was suggested, for example, that a metric should take into account the variations in lowfrequency sound energies since A-weighted sound pressure level measurements cannot properly assess soundscapes across different sound sources (Leventhall 2004), loudness-based parameters neither directly reflect annoyance, nor sufficiently describe soundscapes that human really perceive (Genuit, Fiebig 2006).

Psychoacoustics describing sound perception mechanisms is another important field of the different dimensions involved in the soundscape evaluation process (Genuit, Fiebig 2006). Psychoacoustic parameters are usually used to measure single sound sources, such as vehicle pass-by noise (Genuit, Fiebig 2006). However, soundscapes usual consist of a number of spatially distributed

Corresponding authors: Jiang Liu, Jian Kang

E-mails: river.1.1984@hotmail.com; j.kang@sheffield.ac.uk 
sound sources. Soundscape evaluation is also affected by human hearing characteristics, such as binaural hearing and its consequential directional hearing and selectivity that could classify complex soundscapes into single sound events that are selected by human hearing and decisively influence the individual evaluation. Thus, these psychoacoustic parameters should be used in combination with physical, binaural signal processing as well as cognitive aspects affecting soundscape evaluation (Genuit, Fiebig 2006).

Subjective methods have also been widely used in soundscape studies, in which different sounds or soundscapes were usually perceived or recalled by human raters (Dubois et al. 2006; Jeon et al. 2010; Kang, Zhang 2010; Lavandier, Defréville 2006; Yang, Kang 2005a, 2005b; Yu, Kang 2008), and inquiring opinions through interviews or questionnaires to the individuals concerned are usual approaches (Liu et al. 2013a; Yang, Kang 2005a, 2005b; Yu, Kang 2008). Another typical approach is the semantic difference analysis, which is used to identify the most important factors in evaluating the overall soundscape and individual sounds (Kang, Zhang 2010). It is clear that soundscape perception is a complex process, which could be affected not only by the acoustical features, but also the personal, social and behavioural variables of people (Schulte-Fortkamp 2002; Yu, Kang 2008). The above soundscape parameters could either objectively or subjectively characterise part of soundscapes, and in many cases a combined use of the objective and subjective soundscape information were adopted (De Coensel et al. 2011; Jeon et al. 2010). However, most of these parameters are only effective to characterise existing soundscapes and hard to predict possible soundscape effects, and also tend to characterise them holistically, ignoring the fact that soundscapes are objective compositions of different sound sources with spatial and temporal variability (Liu et al. 2013b).

The soundscape information in relation to the soundscape components could be very important and have practical significance, because it is related to certain sound sources and thus designable, which makes the soundscape intervention/design process more pointed. Therefore, more effective parameters indicating the soundscape composition information, termed in this study as soundscape composition parameters, are of great significance for the soundscape design process. Moreover, as one of the advantages of the physical and psychoacoustic parameters is that they are relatively easily measured, attempting to build relationships between them and soundscape composition information may make them more effective in characterising soundscapes.

In the present study, therefore, a series of soundscape composition parameters is proposed to reveal the "objective" soundscape composition characteristics. With an aim to examine whether and how the soundscape composition parameters are related with the physical and psychoacoustic parameters, a case study based on specifically designed soundwalks was conducted in five city parks in Xiamen, China. The study also attempts to explore the possibility to use various soundscape parameters together to facilitate the soundscape design process.

\section{Methods}

\subsection{Soundscape composition parameters}

Previous research on soundscape composition characteristics have been conducted in both rural and urban areas, where soundscapes were treated as a series of perceptible sounds recognised by a group of pre-trained observers following specifically defined spatiotemporal scales (Liu et al. 2013b; Matsinos et al. 2008). The approach could be categorised as a type of soundwalk, which is usually conducted by a group of people following a pre-defined walking route (or specific sites) and using a structured protocol with a high level of sonic awareness, during which soundscape quality may be evaluated (Kang, Zhang 2010; Schafer 1969). With this kind of approach, the protocols were essentially sound sources identification and loudness/intensity evaluation. Parameters such as perceived loudness of individual sounds/sound categories could provide the "objective" soundscape information.

In a recent study, several parameters reflecting "objective" soundscape information, including perceived loudness and occurrences of individual sound categories and soundscape diversity index was studied in relation to landscape characteristics (Liu et al. 2014). These parameters are soundscape composition parameters, which refer to parameters that could objectively characterise the existence of different sound sources in a soundscape. A basic hypothesis was that, no matter how people perceive the soundscapes, the objective existence of them in terms of composition of different sound sources, or the so-called "objective" soundscape information, does not change. This kind of information, which related to detailed sound source identification could be given by human listeners, but such "objective" aspects of soundscapes would not be affected by personal differences, especially after a pretraining and "calibration" process. The three soundscape composition parameters proposed in the study are defined below (Liu et al. 2014):

(1) Perceived loudness of individual sound or sound category (PLS): the mean of all the perceived loudness scores of a sound provided by a group of observers using the same pre-defined rating scale (five-point linear scale from "very quiet" to "very loud" in this study). PLS of a sound category is the sum of PLS of the corresponding individual sounds within the category. 
(2) Perceived occurrence of individual sound or sound category (POS): the occurrences of a sound recorded in the observation period divided by the observation time-steps, and POS of a sound category is the sum of POS of the corresponding individual sounds within the category.

(3) Soundscape diversity index (SDI): the probability that two individual sounds, randomly selected from a soundscape sample, will belong to different types of sound, which is calculated by:

$$
S D I=1-\sum_{i=1}^{S}\left(\frac{n}{N}\right)^{2},
$$

where $n$ and $N$ are the total number of perceived occurrences of a particular sound $i$ and all $S$ kinds of sound appeared in the soundscape sample, respecti-
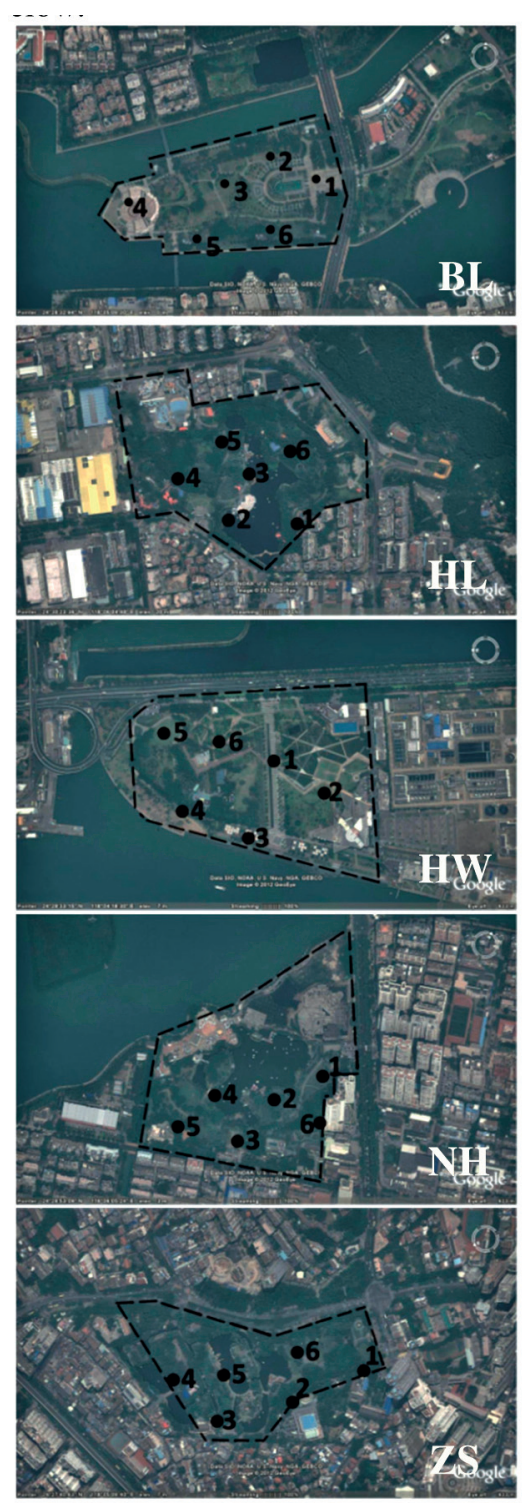

Fig. 1. Images of the five studied city parks from Google Earth, shown with the areas with broken line, BL: Bailuzhou (west), HL: Huli, HW: Haiwan, NH: Nanhu, ZS: Zhongshan vely. SDI ranges between 0 and 1 , and the greater the value, the more diverse the soundscape.

Among the three parameters representing different aspects of the soundscape composition characteristics, PLS and POS indicate the composition of individual sounds/ sound categories, and SDI shows the overall soundscape composition characteristics.

\subsection{Case study sites}

City parks serve as central functional open spaces, and are of a strategic importance for the quality of life of our increasingly urbanised society, especially in many Chinese cities that have high construction density (Chiesura 2004; Thompson 2002). Xiamen, for example, as one of the four earliest areas in China that implemented the openingdoor policies in the 1980s, has become the largest and most important city in south-eastern China with about 3.67 million permanent residents. The city centre is on the Xiamen Island, which has an area of $132.5 \mathrm{~km}^{2}$. City parks in Xiamen, in terms of the number and quality, make the city famous and are important recreation spaces for citizens and tourists. In particular, soundscapes in city parks could be a sensitive factor affecting visitors' satisfaction degree (Liu et al. 2013a).

The survey was carried out in five public city parks in the city centre of Xiamen, namely Bailuzhou (west), Huli, Haiwan, Nanhu and Zhongshan. The satellite images of these parks from Google earth are shown in Figure 1. These parks were chosen because they have comparable characteristics in terms of location, scale, function and public importance, according to the list of the Xiamen Construction and Administration Bureau, and together they could represent diverse sample sites with different landscape situations.

In terms of the sound sources in the parks, based on several pilot investigations, eighteen regularly appearing sounds were identified, which were classified into five sound categories, including human, traffic, mechanical, biological and geophysical sounds, as shown in Table 1, as a reference for the observers during the soundwalks as discussed below.

\subsection{Soundwalks}

The soundwalks in this study involved seven observers with normal hearing abilities ( 4 female and 3 male, average age $25 \pm 1.5$ years). Before performing the soundwalks, they all went through a training process, including being familiar with all the major sounds and their codes to ensure a fast recording; and performing pilot studies to learn the investigation process and minimise recording bias. The aim of the training process is also to minimize the influence of subjective factors of the observers, including these from the cultural background, and only their ability 
to perceive and record the sounds is concerned. On-site soundwalks were conducted in June 2012 in each of the five parks in five consecutive workdays with stable weather conditions, respectively.

In each park, six sampled sites were evenly chosen along the main visitor paths and consecutively numbered as a sequence of the soundwalk route, as shown in Figure 1. The soundwalks were conducted in three different periods of a day in each park, namely morning (07:0009:00), afternoon (12:00-14:00), and dusk (17:00-19:00), respectively, in order to minimise the timing effect and to get enough sample data for statistical analysis. Within each period, all six sampled sites were visited once following the same sequence. At each site, the codes of the heard sounds were entered into a table in 5 minute intervals which was further divided into ten sequential time-steps of 30 second each. Within each time-step, the perceived loudness of each individual sound was scored on a five-point linear scale $(1=$ very quiet, $2=$ quiet, $3=$ normal, $4=$ loud, $5=$ very loud). At the same time, during subjective recording of soundscape data, the 5-minute soundscapes were recorded using a Sony PCM-D50 sound recorder with a tripod at a height of $1.2 \mathrm{~m}$ (binaural, sampled at $44.1 \mathrm{kHz}$ with a 16-bit depth).

Table 1. Recognised sounds and their corresponding sound categories in the city parks

\begin{tabular}{llc}
\hline Sound category & \multicolumn{1}{c}{ Sound } & Code \\
\hline \multirow{3}{*}{$\begin{array}{l}\text { Human sound } \\
\text { (Hum) }\end{array}$} & Surrounding speech & SS \\
& Children shouting & CS \\
& Footsteps & FS \\
& Exercising & EX \\
\hline Traffic sound & Traffic sound & TS \\
(Traf) & Bicycle riding & BR \\
\hline \multirow{4}{*}{ Mechanical } & Entertainment facilities & EF \\
sound (Mech) & Andistinguishable sound & AF \\
& Lawn mowing & OS \\
& Road cleaning & LM \\
& Music & RC \\
& Birds & MS \\
\hline \multirow{2}{*}{ Biological sound } \\
(Bio) & Dogs & BS \\
& Insects & DB \\
Geophysical & Water sound & IS \\
sound (Geo) & Leaves rustling & WS \\
& Wind & LR \\
\hline & & WB \\
\hline
\end{tabular}

In order to verify whether all the seven observers were recognising a similar set of sounds (occurrences) and using similar evaluation scales (loudness) within a certain period, inter-rater reliability of the seven observers for perceived occurrence and loudness of each of the five major sound categories was analysed using Cronbach's alpha method. The result of a mean inter-rater reliability of $0.94 \pm 0.03$ and $0.96 \pm 0.02$ for POS and PLS, respectively, could guarantee the suitability of processing the soundscape data sets from all protocols by the seven observers into one. During the data process, only the sounds recorded by more than 3 participants in the same time-steps were regarded as effective recordings in each soundscape recording, in order to get a more reliable and "objective" data set. To simplify the analysis process, PLS and POS were analysed only for the five major sound categories, namely human, traffic, mechanical, biological and geophysical sounds.

\subsection{Physical and psychoacoustic parameters}

In terms of physical parameters, in addition to the conventional sound pressure levels, specifically $\mathrm{L}_{\mathrm{Aeq}}$ and $\mathrm{L}_{\mathrm{A} 10}$ (the noise level just exceeded for $10 \%$ of the measurement period), parameters like $\mathrm{L}_{\mathrm{A} 10}-\mathrm{L}_{\mathrm{A} 90}$ and $\mathrm{L}_{\mathrm{Ceq}}-\mathrm{L}_{\text {Aeq }}$, indicating fluctuation difference and $\mathrm{dB}$ change at low frequencies, respectively, were also introduced to characterise acoustic features that may be closer to human perception (De Coensel, Botteldooren 2006; De Coensel et al. 2011; Rychtáriková, Vermeir 2013). Although the effectiveness of psychoacoustic parameters on measuring environmental sounds with multiple sound sources is debatable, they are included mainly because the "descriptive listening" of the soundwalk approach is related to perception of individual sound sources (Raimbault 2006). Frequently used psychoacoustic parameters including loudness, sharpness, roughness, fluctuation strength, and speech intelligibility were chosen in the study (Hall et al. 2013; Rychtáriková, Vermeir 2013).

Physical parameters including $\mathrm{L}_{\text {Aeq, }}, \mathrm{L}_{\mathrm{Ceq}}, \mathrm{L}_{\mathrm{A} 10}$ and $\mathrm{L}_{\mathrm{A} 90}$, and the psychoacoustic parameters mentioned above were calculated for each sampled site in each period and generated 89 data samples (one sound recording at site 4 during the afternoon period in Nanhu park was lost). All the parameter values were the energetic average between the left and right channel. $\mathrm{L}_{\mathrm{A} 10}-\mathrm{L}_{\mathrm{A} 90}$ and $\mathrm{L}_{\mathrm{Ceq}}-\mathrm{L}_{\text {Aeq }}$ were calculated accordingly.

\subsection{Statistical analysis}

Factor analysis examines the underlying (or latent) relationships among the variables. It was conducted to find out the structure of all the soundscape parameters. Since all the soundscape parameters were measured by scale, Pearson correlations were carried out to analyse the relationships among the soundscape composition parameters, and between each of the soundscape composition parameters and the physical and psychoacoustic parameters. Stepwise multiple regressions were also performed to 
reveal the relationships among the soundscape composition parameters and the physical and psychoacoustic parameters. All the statistical analyses were conducted using SPSS 16.0 software.

\section{Results and discussion}

\subsection{Necessity to introduce the soundscape composition parameters}

To introduce new parameters into the soundscape evaluation/characterisation approach, it is important to test whether these parameters could properly characterise a soundscape and present more information than the existing parameters. Thus, firstly the values of the soundscape composition parameters were related to the SPL and spectrum of the same soundscape recording from the recorder. As an example, site No. 3 in Huli park during the afternoon period was chosen because of the diverse sound sources and fluctuating SPL, as shown in Figure 2. Combined with playback of the sound recording, it is demonstrated that there were mainly four different kinds of sound that are spectrally separated in the spectrum chart, including traffic sounds (about 25-200 Hz) during the whole recording period, human sounds (about 500-2k Hz) appearing irregularly and sometimes with high SPL (child shouting, surrounding speech, footsteps), biological sounds including regularly appearing birdsongs (about $1.5 \mathrm{k}-2 \mathrm{k} \mathrm{Hz}$ ) during the whole period and sound from insects with the highest frequency (about 3k-10k Hz), irregular but with four lasting periods. All the sounds were also recognised and recorded by the observers during the soundwalk.

As also shown in Figure 2, recognised sound categories from sound recording playback were noted in the order

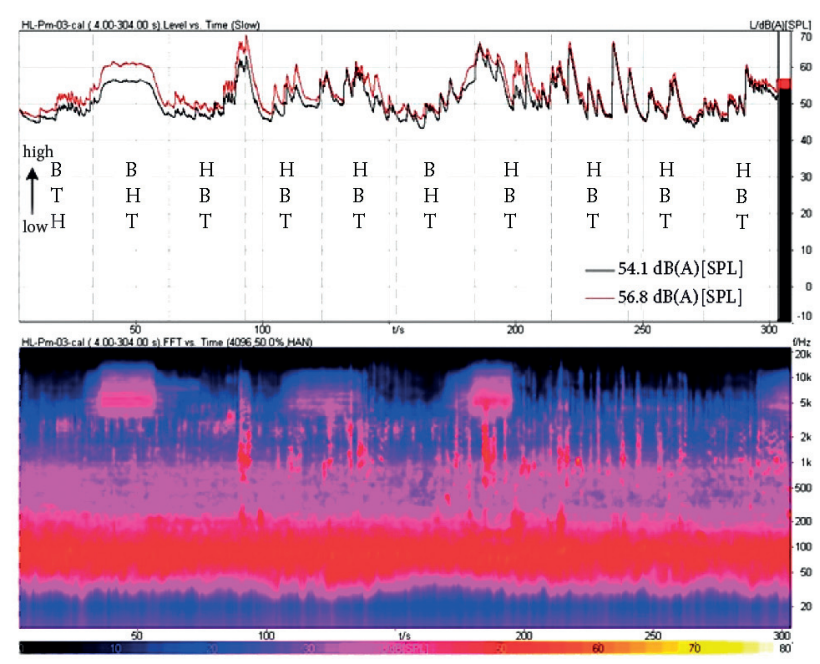

Fig. 2. SPL and spectrum change on site No. 3 in Huli park in the afternoon period, together with recognised sound categories by playback of the sound recording in the order from high to low perceived loudness, $\mathrm{H}$ : human sounds, B: biological sounds, T: traffic sounds from high to low perceived loudness for each of the same 30 s time-steps as during the soundwalk, and the results were highly comparable to those of the soundwalk (7 out of 10 were exactly the same). It showed that loudness perception of different sound category was not always consistent with its SPL. For example, traffic sounds as background sounds contributed the most to the SPL of the soundscape, but they were not evaluated higher than the foreground human and biological sounds. Moreover, mechanical sound (music) recognised through the soundwalk was hard to detect by sound recording playback. To recapitulate, the on-site subjective recording during soundwalks has advantages in characterising a soundscape, and may supply more information than only playback of the sound recording and the subsequent analysis.

Factor analysis of all the soundscape parameters was performed using all the data from the five city parks. Varimax rotated principal component analysis was employed to extract the orthogonal factor underlying the 20 soundscape parameters. With a criterion factor of eigenvalue $>1$, five factors were determined, as shown in Table 2 .

Table 2. Factor analysis of all the soundscape parameters (overall results of all the five city parks), cumulative \%: 78; extraction method: principal component analysis; rotation method: varimax with Kaiser normalisation; $\mathrm{N}=89$

\begin{tabular}{|c|c|c|c|c|c|c|}
\hline \multirow{2}{*}{\multicolumn{2}{|c|}{$\begin{array}{c}\text { Sound } \\
\text { parameters }\end{array}$}} & \multicolumn{5}{|c|}{ Factor } \\
\hline & & $1(30 \%)$ & $2(15 \%)$ & $3(11 \%)$ & $4(11 \%)$ & $5(11 \%)$ \\
\hline \multirow{5}{*}{ PLS } & Hum & 0.473 & 0.001 & -0.197 & 0.739 & -0.272 \\
\hline & Traf & 0.115 & -0.791 & -0.081 & -0.237 & -0.004 \\
\hline & Mech & 0.101 & 0.801 & -0.222 & -0.146 & -0.389 \\
\hline & Bio & -0.054 & -0.215 & -0.107 & -0.190 & 0.861 \\
\hline & Geo & -0.030 & -0.069 & 0.928 & 0.010 & -0.048 \\
\hline \multirow{5}{*}{ POS } & Hum & 0.308 & -0.039 & -0.291 & 0.821 & -0.255 \\
\hline & Traf & -0.124 & -0.826 & 0.036 & -0.069 & 0.011 \\
\hline & Mech & 0.101 & 0.801 & -0.222 & -0.146 & -0.389 \\
\hline & Bio & -0.108 & -0.219 & 0.022 & -0.161 & 0.749 \\
\hline & Geo & -0.197 & -0.147 & 0.903 & 0.001 & -0.004 \\
\hline \multicolumn{2}{|l|}{ SDI } & 0.004 & 0.097 & 0.105 & 0.788 & -0.097 \\
\hline \multicolumn{2}{|l|}{$\mathrm{L}_{\text {Aeq }}$} & 0.945 & 0.072 & -0.125 & 0.103 & -0.045 \\
\hline \multicolumn{2}{|l|}{$\mathrm{L}_{\mathrm{A} 10}$} & 0.958 & 0.102 & -0.151 & 0.115 & -0.012 \\
\hline \multicolumn{2}{|c|}{$\mathrm{L}_{\mathrm{A} 10}-\mathrm{L}_{\mathrm{A} 90}$} & 0.317 & 0.456 & -0.199 & 0.123 & 0.460 \\
\hline \multicolumn{2}{|c|}{$\mathrm{L}_{\mathrm{Ceq}}-\mathrm{L}_{\mathrm{Aeq}}$} & -0.592 & -0.151 & 0.440 & -0.237 & -0.129 \\
\hline \multicolumn{2}{|c|}{ Loudness } & 0.933 & -0.003 & -0.047 & 0.057 & -0.079 \\
\hline \multicolumn{2}{|c|}{ Sharpness } & 0.788 & 0.284 & 0.034 & -0.038 & 0.212 \\
\hline \multicolumn{2}{|c|}{ Roughness } & 0.945 & -0.067 & -0.043 & 0.064 & -0.141 \\
\hline \multicolumn{2}{|c|}{$\begin{array}{l}\text { Fluctuation } \\
\text { strength }\end{array}$} & 0.103 & -0.037 & -0.183 & -0.350 & -0.235 \\
\hline \multicolumn{2}{|c|}{$\begin{array}{l}\text { Speech } \\
\text { intelligi- } \\
\text { bility }\end{array}$} & -0.954 & 0.027 & 0.087 & -0.042 & 0.182 \\
\hline
\end{tabular}


It can be seen that the five factors cover $78 \%$ of the total variance. Factor $1(30 \%)$ is mainly associated with most of the physical and psychoacoustic parameters, including $\mathrm{L}_{\text {Aeq }}, \mathrm{L}_{\mathrm{A} 10}, \mathrm{~L}_{\mathrm{Ceq}}-\mathrm{L}_{\text {Aeq }}$, loudness, sharpness, roughness and speech intelligibility. Factor 2 (15\%) is generally associated with perception of traffic and mechanical sounds, including PLS and POS of both traffic and mechanical sounds and $\mathrm{L}_{\mathrm{A} 10}-\mathrm{L}_{\mathrm{A} 90}$. Factor $3(11 \%)$ is principally related to perception of geophysical sounds, including both PLS and POS of geophysical sounds. Factor 4 (11\%) is mostly associated with perception of human sounds, including both PLS and POS of human sounds, SDI and fluctuation strength. Factor $5(11 \%)$ is mainly associated with perception of biological sounds, including POS of biological sounds.

It is noted that, while the physical and psychoacoustic parameters cluster mostly in factor 1 , the soundscape composition parameters almost dominate all the other four factors. In particular, the soundscape composition parameters of individual sound categories play important roles. Overall, the soundscape composition parameters could provide much new information which the other physical and psychoacoustic parameters could not. Consequently, the application of the soundscape composition parameters should be examined in depth, as well as considering their relationships with physical and psychoacoustic parameters.

\subsection{Relationships among the soundscape composition parameters}

Figure 3 shows both PLS and POS of individual sound categories in summed value of all sampled sites in each period in different city parks. It is clear that the soundscape compositions of these parks characterised by these parameters were generally different. The dominant sounds (percentage $>30 \%$ ) varied in different periods in each park. In terms of PLS, human sounds were the most frequently perceived sounds in these parks, dominating 8 out of the 15 site-periods, followed by biological and mechanical sounds, each dominating 4 and 3 site-periods, respectively. In terms of POS, human sounds dominated 7 out of the 15 site-periods, while mechanical and biological sounds dominated 5 and 4 site-periods, respectively.

Correlations among the soundscape composition parameters of the five sound categories indicate that PLS and POS of the same individual sound category were all positively correlated for the five categories. PLS or POS of certain sound category was generally negatively correlated with that of the other sound categories if significant relationships existing. For example, human and mechanical sounds were only negatively related to biological and geophysical sounds, respectively. However, the parameters of traffic sounds and biological sounds were all positively

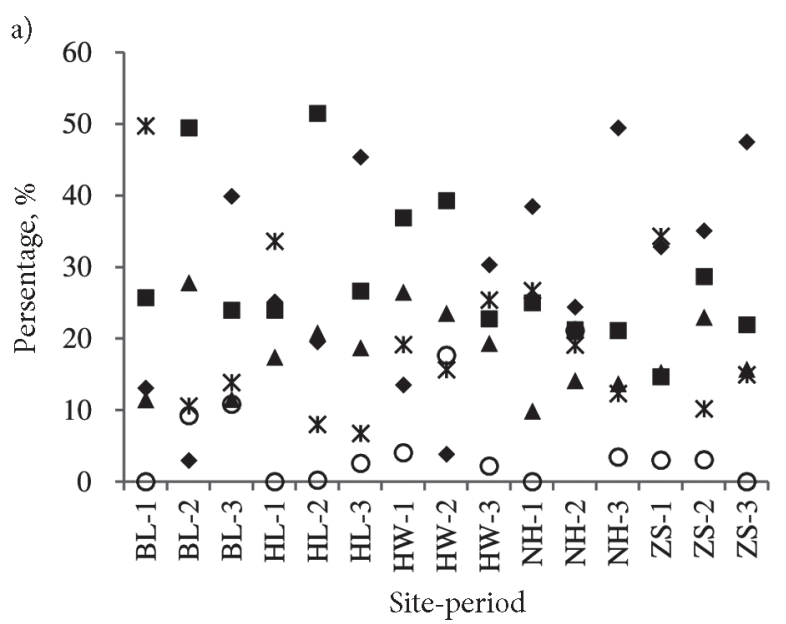

-PLS_Hum \ PLS_Traf XPLS_Mech $\square$ PLS_Bio OPLS_Geo

b)

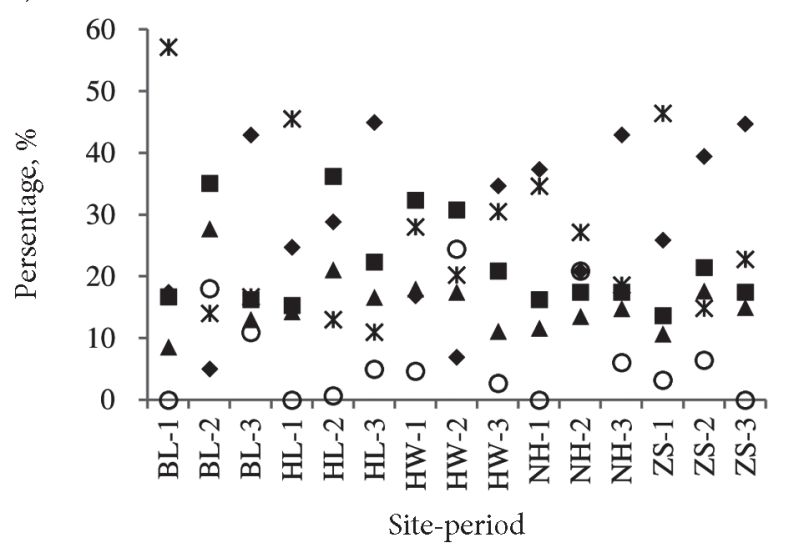

-POS_Hum \ POS_Traf $\boldsymbol{*}$ POS_Mech $\square$ POS_Bio OPOS_Geo

Fig. 3. Perceived loudness (a) and occurrences (b) of individual sound categories in each city park per period (sum of all sampled sites)

correlated. The results also show that SDI was positively related to both PLS (0.504) and POS (0.612) of human sounds, and negatively related to PLS of biological sounds $(-0.22)$. It indicates that soundscape elements in the city parks were normally dominated by human sounds, and the perception of biological sounds might be impaired in this situation.

Overall, the above analysis show that the differences among different soundscapes could be well characterised by the soundscape composition parameters. Their inter relationships indicate how different sounds could affect each other in the composition of the soundscapes, and therefore, provide an insight as to how the other sounds will be affected, when changing one or more of the soundscape components.

\subsection{Relationships between the soundscape composition parameters and the physical parameters}

General correlation relationships between the soundscape composition parameters of individual sound categories 


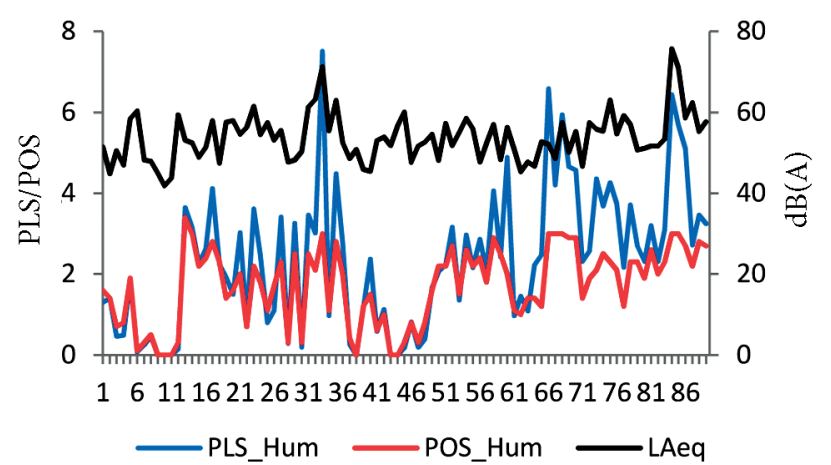

Fig. 4. Perceived loudness and occurrences of human sounds along with sound pressure level $\left(\mathrm{L}_{\text {Aeq }}\right)$ at each sampled site during each period, BL: 1-18, HL: 19-36, HW: 37-54, NH: 55-71, ZS: 72-89

and the four physical parameters could reveal the sound type that could affect the physical characteristics of soundscapes. Pearson correlation analysis results between each of the soundscape composition parameters and the physical parameters show that (correlation coefficient are shown in the bracket respectively), PLS and POS of human sounds were both positively related to $\mathrm{L}_{\text {Aeq }}(0.525,0.387)$ and $\mathrm{L}_{\mathrm{A} 10}(0.552,0.426)$, and negatively related to $\mathrm{L}_{\mathrm{Ceq}}-\mathrm{L}_{\text {Aeq }}(-0.542,-0.448)$. PLS and POS of traffic sounds were both negatively related to $\mathrm{L}_{\mathrm{A} 10}-\mathrm{L}_{\mathrm{A} 90}$ $(-0.265,-0.293)$. POS of geophysical sounds was significantly correlated to all the four physical parameters, while no soundscape composition parameter of mechanical or biological sounds or SDI showed significant relationships with the physical parameters. The results indicate that human sounds were the key factors affecting sound levels in city parks as they were the only sound type positively related to $\mathrm{L}_{\text {Aeq }}$ and $\mathrm{L}_{\mathrm{A} 10}$. This could be more clearly observed in Figure 4, in which the values of PLS and POS of human sounds and $\mathrm{L}_{\text {Aeq }}$ indicating similar trends are shown for all the 89 soundscape samples. Excessive human sounds could impair the sound level changes at low frequencies. Traffic sounds had more effects on sound level fluctuation differences. With more traffic sounds, the SPL fluctuation is lower. Geophysical sounds were the only sound type showing positive relationships with the sound level change in low frequencies. It is also noticed that, the physical parameters showed no relationship with mechanical or biological sounds, and the same was true with SDI. This further suggests the necessity to introduce the soundscape composition parameters.

Stepwise regression analysis was conducted between each of the soundscape composition parameters and the physical and psychoacoustic parameters, to test whether and to what extent the dependent variable could be explained by the independent variables. In terms of the physical parameters as independent variables, as shown in Table 3, PLS of human sounds could be explained by $\mathrm{L}_{\mathrm{A} 10}, \mathrm{~L}_{\mathrm{Ceq}}-\mathrm{L}_{\text {Aeq }}$ and $\mathrm{L}_{\mathrm{A} 10}-\mathrm{L}_{\mathrm{A} 90} . \mathrm{L}_{\mathrm{Ceq}}-\mathrm{L}_{\text {Aeq }}$ was the only physical parameter related to POS of human sounds. PLS and POS of traffic sounds could only be explained by $\mathrm{L}_{\mathrm{A} 10}-\mathrm{L}_{\mathrm{A} 90}$, and PLS and POS of geophysical sounds could only be explained by $\mathrm{L}_{\mathrm{Ceq}}-\mathrm{L}_{\text {Aeq }}$. No physical parameter could explain PLS and POS of mechanical or biological sounds or SDI. $\mathrm{L}_{\text {Aeq }}$ was not suitable as an explanatory variable for all the soundscape composition parameters, which resonates with the ineffectiveness of single sound level measurements of soundscapes (Raimbault, Dubois 2005). As expected, $\mathrm{L}_{\mathrm{A} 10}-\mathrm{L}_{\mathrm{A} 90}$ and $\mathrm{L}_{\mathrm{Ceq}}-\mathrm{L}_{\text {Aeq }}$ showed the closest relationships with soundscape composition parameters of certain sound categories, including human, traffic and geophysical sounds, which means they are more suited to be used as soundscape characterisation parameters. It is also noted that, although the regressions are all significant, the adjusted $\mathrm{R}^{2}$ values of them are mostly low. This means the explanatory ability of the physical parameters to the soundscape composition parameters is limited.

Conversely, whether and to what extent the physical parameters could be explained by the soundscape composition parameters were also tested by stepwise regression analysis. The results are shown in Table 4. It can be seen that $\mathrm{L}_{\text {Aeq }}$ in the city parks could be explained by PLS of human sounds and SDI, which again shows that human sounds were the key factors affecting the sound levels in city parks. $\mathrm{L}_{\mathrm{A} 10}$ could be explained by both PLS and POS of human sounds and POS of geophysical sounds. There were three soundscape composition parameters introduced as explanatory variables for $\mathrm{L}_{\mathrm{A} 10}-\mathrm{L}_{\mathrm{A} 90}$, i.e., POS of both traffic and geophysical sounds and PLS of biological sounds. $\mathrm{L}_{\mathrm{Ceq}}-\mathrm{L}_{\text {Aeq }}$ was best explained by four soundscape composition parameters, i.e., PLS of human and biological sounds, POS of mechanical sounds and SDI. By comparing the adjusted $\mathrm{R}^{2}$ values of these regressions, it is obvious that the soundscape composition parameters showed more explanatory ability to physical parameters than reversed.

Overall, soundscape composition parameters of human, traffic and geophysical sounds were all related to and could be explained by certain physical parameters. However, for soundscape composition parameters of mechanical and biological sounds, neither of the two cases was true. The results indicate again the necessity to introduce soundscape composition parameters, especially for the sounds that are hard to characterise by physical parameters. Soundscape composition parameters from all the five types of individual sound categories as well as SDI showed explanatory ability to some specific physical parameters as described above. 
Table 3. Regression analysis results between each of the soundscape composition parameters and the physical and psychoacoustic parameters, where no relationship was shown are marked with "—”; ${ }^{\star} p<0.05,{ }^{\star *} p<0.01$

\begin{tabular}{|c|c|c|c|c|c|c|}
\hline & Dependent variable & Variables & $\beta$ & $\mathrm{t}$ & Adjusted $\mathrm{R}^{2}$ & $\mathrm{~F}$ \\
\hline \multirow{5}{*}{ PLS } & Hum & $\begin{array}{l}\mathrm{L}_{\mathrm{A} 10} \\
\mathrm{~L}_{\mathrm{Ceq}}-\mathrm{L}_{\text {Aeq }} \\
\mathrm{L}_{\mathrm{A} 10}-\mathrm{L}_{\mathrm{A} 90} \\
\text { Sharpness }\end{array}$ & $\begin{array}{c}0.572 \\
-0.439 \\
-0.253 \\
-0.253\end{array}$ & $\begin{array}{l}4.458^{\star *} \\
-4.035^{\star *} \\
-2.717^{\star *} \\
-2.139^{\star}\end{array}$ & 0.424 & $17.179^{* x}$ \\
\hline & Traf & $\mathrm{L}_{\mathrm{A} 10}-\mathrm{L}_{\mathrm{A} 90}$ & -0.265 & $-2.567^{\star}$ & 0.06 & $6.591^{\star}$ \\
\hline & Mech & Sharpness & 0.249 & $2.395^{\star}$ & 0.051 & $5.737^{\star}$ \\
\hline & Bio & - & - & - & - & - \\
\hline & Geo & $\begin{array}{l}\mathrm{L}_{\mathrm{Ceq}}-\mathrm{L}_{\text {Aeq }} \\
\text { Sharpness }\end{array}$ & $\begin{array}{l}0.506 \\
0.281 \\
\end{array}$ & $\begin{array}{c}4.319^{\star *} \\
2.398^{\star} \\
\end{array}$ & 0.159 & $9.325^{\star *}$ \\
\hline \multirow{5}{*}{ POS } & Hum & $\begin{array}{l}\mathrm{L}_{\mathrm{Ceq}}-\mathrm{L}_{\text {Aeq }} \\
\text { Roughness } \\
\text { Sharpness }\end{array}$ & $\begin{array}{c}-0.422 \\
0.426 \\
-0.339\end{array}$ & $\begin{array}{c}-3.798^{\star *} \\
3.329^{\star *} \\
-2.546^{\star}\end{array}$ & 0.272 & $11.981^{* *}$ \\
\hline & Traf & $\mathrm{L}_{\mathrm{A} 10}-\mathrm{L}_{\mathrm{A} 90}$ & -0.293 & $-2.854^{\star *}$ & 0.075 & $8.147^{\star \star}$ \\
\hline & Mech & Sharpness & 0.249 & $2.395^{*}$ & 0.051 & $5.737^{\star}$ \\
\hline & Bio & - & - & - & - & - \\
\hline & Geo & $\mathrm{L}_{\mathrm{Ceq}}-\mathrm{L}_{\text {Aeq }}$ & 0.438 & $4.545^{\star \star}$ & 0.183 & $20.653^{* *}$ \\
\hline SDI & & - & - & - & - & - \\
\hline
\end{tabular}

Table 4. Regression analysis results between each of the physical and psychoacoustic parameters and soundscape composition parameters and, where no relationship was shown are marked with "-"; ${ }^{*} p<0.05,{ }^{* *} p<0.01$

\begin{tabular}{|c|c|c|c|c|c|}
\hline Dependent variable & Variables & $\beta$ & $\mathrm{t}$ & Adjusted $\mathrm{R}^{2}$ & $\mathrm{~F}$ \\
\hline \multirow{2}{*}{$\mathrm{L}_{\text {Aeq }}$} & PLS_Hum & 0.633 & $6.099^{\star \star}$ & \multirow{2}{*}{0.294} & \multirow{2}{*}{$19.287^{\star x}$} \\
\hline & SDI & -0.214 & $-2.064^{*}$ & & \\
\hline \multirow{3}{*}{$\mathrm{L}_{\mathrm{A} 10}$} & PLS_Hum & 0.841 & $4.566^{\star *}$ & \multirow{3}{*}{0.36} & \multirow{3}{*}{$17.493^{\star *}$} \\
\hline & POS_Geo & -0.255 & $-2.832^{\star \star}$ & & \\
\hline & POS_Hum & -0.4 & $-2.132^{\star}$ & & \\
\hline \multirow{3}{*}{$\mathrm{L}_{\mathrm{A} 10}-\mathrm{L}_{\mathrm{A} 90}$} & POS_Traf & -0.293 & $-2.841^{\star *}$ & \multirow{3}{*}{0.153} & \multirow{3}{*}{$6.305^{* *}$} \\
\hline & POS_Geo & -0.221 & $-2.193^{\star}$ & & \\
\hline & PLS_Bio & 0.213 & $2.111^{*}$ & & \\
\hline \multirow{4}{*}{$\mathrm{L}_{\mathrm{Ceq}}-\mathrm{L}_{\text {Aeq }}$} & PLS_Hum & -0.821 & $-9.158^{\star \star}$ & \multirow{4}{*}{0.52} & \multirow{4}{*}{$24.859^{* *}$} \\
\hline & PLS_Bio & -0.468 & $-5.388^{\star \star}$ & & \\
\hline & POS_Mech & -0.376 & $-4.587^{\star \star}$ & & \\
\hline & SDI & 0.287 & $3.34^{* *}$ & & \\
\hline \multirow{2}{*}{ Loudness } & PLS_Hum & 0.966 & $5.005^{\star *}$ & \multirow{2}{*}{0.294} & \multirow{2}{*}{$19.336^{\star x}$} \\
\hline & POS_Hum & -0.525 & $-2.721^{\star \star}$ & & \\
\hline \multirow{5}{*}{ Sharpness } & PLS_Hum & 0.673 & $6.469^{* *}$ & \multirow{5}{*}{0.412} & \multirow{5}{*}{$13.322^{\star x}$} \\
\hline & PLS_Bio & 0.597 & $5.907^{* *}$ & & \\
\hline & PLS_Mech & 0.567 & $5.827^{\star \star}$ & & \\
\hline & PLS_Geo & 0.32 & $3.513^{* *}$ & & \\
\hline & SDI & -0.31 & $-3.193^{\star *}$ & & \\
\hline \multirow{4}{*}{ Roughness } & PLS_Hum & 0.666 & $6.853^{* *}$ & \multirow{4}{*}{0.392} & \multirow{4}{*}{$15.203^{\star x}$} \\
\hline & PLS_Traf & 0.399 & $3.606^{* *}$ & & \\
\hline & POS_Traf & -0.291 & $-2.631^{\star \star}$ & & \\
\hline & SDI & -0.251 & $-2.597^{\star}$ & & \\
\hline Fluctuation strength & - & - & - & - & - \\
\hline \multirow{4}{*}{ Speech intelligibility } & PLS_Hum & -0.723 & $-8.049^{\star *}$ & \multirow{4}{*}{0.465} & \multirow{4}{*}{$20.098^{\star x}$} \\
\hline & PLS_Traf & -0.357 & $-4.071^{\star \star}$ & & \\
\hline & PLS_Mech & -0.323 & $-3.702^{\star \star}$ & & \\
\hline & SDI & 0.295 & $3.249^{\star *}$ & & \\
\hline
\end{tabular}




\subsection{Relationships between the soundscape composition parameters and the psychoacoustic parameters}

Pearson correlation analysis results between each of the soundscape composition parameters and the psychoacoustic parameters show that, fluctuation strength was not related to any of the soundscape composition parameters. Human sounds showed the most significant relationships with the psychoacoustic parameters. PLS and POS of human sounds were all significantly and positively related to loudness $(0.501,0.331)$ and roughness $(0.521,0.403)$, and negatively related to speech intelligibility $(-0.551$, $-0.399)$. PLS of human sounds was also positively related to sharpness $(0.305)$. The results suggest that more human sounds increased the loudness and roughness perception of soundscapes in the parks, and impaired the speech intelligibility. For traffic sounds, only negative relationships existed between POS of traffic sounds and sharpness $(-0.251)$. Sharpness was also the only parameter which was positively related to both PLS and POS of mechanical sounds $(0.249,0.249)$. The results suggest that frequently perception of traffic sounds could minimize sharpness perception of the soundscapes, while existence of excessive mechanical sounds could be the major cause of more sharpness perception of the soundscapes. Although PLS of geophysical sounds showed no relationship with any of the psychoacoustic parameters, which was mainly because of the low loudness levels of these kinds of sound compared with those of other sounds in the parks (Fig. 3), POS of geophysical sounds showed relationships with three of the psychoacoustic parameters, namely, negatively correlated with loudness $(-0.245)$ and roughness $(-0.241)$, and positively with speech intelligibility $(0.271)$, which means this type of sound could mitigate the perceived loudness and roughness of soundscapes and improve the speech intelligibility. PLS and POS of biological sounds showed no relationship with any of the psychoacoustic parameters, and the results were the same with SDI.

The regression analysis results showing the explanatory ability of the psychoacoustic parameters to the soundscape composition parameters are shown in Table 3. It can be seen that, sharpness was almost the only variable related to the soundscape composition parameters of individual sound categories. It showed negative relationships with both PLS and POS of human sounds and positive relationships with both PLS and POS of mechanical sounds, respectively, as well as positive relationship with PLS of geophysical sounds. Roughness was another parameter as an explanatory variable of POS of human sounds. Neither PLS nor POS of biological sounds could be explained by any of the psychoacoustic parameters. The psychoacoustic parameters showed ineffectiveness in explaining SDI too. As indicated by the low adjusted $\mathrm{R}^{2}$ values of all these regressions, it is clear that the explanatory ability of the psychoacoustic parameters to the soundscape composition parameters was also limited.

As for the explanatory ability of the soundscape composition parameters to the psychoacoustic parameters, the results in Table 4 show that, loudness could be explained by PLS and POS of human sounds. Sharpness could be explained by five soundscape composition parameters totally, including SDI and PLS of almost all individual sound categories except traffic sounds. PLS and POS of traffic sounds, together with PLS of human sounds and SDI could be regarded as explanatory variables of roughness. There was no soundscape composition parameter which could explain fluctuation strength. Speech intelligibility, depending on the level and the frequency of background noise and the speech spectrum itself, could be explained by four variables, i.e., PLS of human, traffic and mechanical sounds, and SDI. In terms of the adjusted $\mathrm{R}^{2}$ values of all these regressions, psychoacoustic parameters were usually better explained by the soundscape composition parameters, further indicating the importance of the soundscape composition parameters.

Overall, soundscape composition parameters of all individual sound categories except for biological sounds are related to certain psychoacoustic parameters except for fluctuation strength. However, while only sharpness and roughness showed explanatory ability to human and/or mechanical sounds, soundscape composition parameters of all the five kinds of individual sound categories as well as SDI showed explanatory ability to certain psychoacoustic parameters.

Through the regression analysis between the soundscape composition parameters and the physical and psychoacoustic parameters (Table 3 and Table 4), it is also found that some of them were mutually explainable (major positive or negative relationship marked with + or - in the bracket), including PLS of human sounds with $\mathrm{L}_{\mathrm{A} 10}$ $(+), \mathrm{L}_{\mathrm{Ceq}}-\mathrm{L}_{\text {Aeq }}(-)$ and sharpness $(+)$, respectively, POS of traffic sounds with $\mathrm{L}_{\mathrm{A} 10}(-)$, PLS of both mechanical and geophysical sounds with sharpness $(+)$. Their relationships provide reliable information about which types of sound could affect the values of physical and psychoacoustic parameters in city parks, which is instructive for the soundscape design of specific sounds in city parks.

\subsection{Application of the soundscape composition parameters}

Physical and psychoacoustic parameters have been verified that their limited effectiveness in characterising soundscape quality may due to the personal difference of listeners' rating standard (Hall et al. 2013). However, with a controlled soundscape perception process and the proposed soundscape composition parameters, these 
parameters were found in relation to certain physical and psychoacoustic parameters. Their relationships could facilitate the soundscape design process. The soundscape composition parameters could be used together with other physical and psychoacoustic parameters to supply detailed information, which, for example, is more useful when handling soundscapes by changing their compositions. As also shown in this study, while the soundscape composition parameters of biological sounds and SDI showed totally no significant relationship with any of the physical or psychoacoustic parameters and could also not be explained by them, they could be the explanatory variable for several physical and psychoacoustic parameters. In this case, they should always be included as soundscape evaluation parameters upon further tests.

In conclusion, soundscape composition parameters could not only indicate much new soundscape information, which may be crucial to soundscape perception, but also provide more detailed information to explain the connotations of certain physical and psychoacoustic parameters. Thus, soundscapes with certain physical and psychoacoustic characteristics could be designed by considering different sound compositions. Of course, it has to be noted that these relationships may only exist in city parks of a similar type as examined in the case study sites, generally with a normal sound pressure level of 40 to $80 \mathrm{~dB}$ (A) (Fig. 4). For other types of space more studies should be conducted using similar method as in this study.

\section{Conclusions}

In this study, based on a specifically designed soundwalk method in five city parks in Xiamen, China, the relationships among the proposed soundscape composition parameters, including the perceived loudness and occurrences of five individual sound categories, i.e., human, traffic, mechanical, biological and geophysical sounds (PLS, POS) and soundscape diversity index (SDI), and some of the physical and psychoacoustic parameters were analysed.

1. The soundscape composition parameters, especially those of individual sound categories, are the major principle components in characterising soundscapes by factor analysis.

2. PLS and POS of the same individual sound categories are all positively correlated, while PLS or POS of certain sound category are generally negatively correlated with those of the other sound categories. SDI differences in the city parks are attributed to human sounds, and higher SDI values impair the perception of biological sounds.

3. The soundscape composition parameters of human sounds show the strongest relationships with the physical and psychoacoustic parameters, followed by geophysical sounds. However, neither PLS or POS of biological sounds nor SDI shows relationship with them. Further more, $\mathrm{L}_{\mathrm{Ceq}}-\mathrm{L}_{\mathrm{Aeq}}, \mathrm{L}_{\mathrm{A} 10}-\mathrm{L}_{\mathrm{A} 90}$ and sharpness have more explanatory power to the soundscape composition parameters of individual sound categories than other physical and psychoacoustic parameters.

4. The explanatory abilities of the physical and psychoacoustic parameters to the soundscape composition parameters are all limited, whereas the soundscape composition parameters show more explanatory abilities to them. PLS of human sounds is the most frequently introduced variable for nearly all the other objective parameters except $\mathrm{L}_{\mathrm{A} 10}-\mathrm{L}_{\mathrm{A} 90}$ and fluctuation strength, followed by SDI explaining five objective soundscape parameters.

5. The relationships between all the soundscape parameters that are mutually explainable indicate more concrete and reliable information in terms of explaining the physical and psychoacoustic parameters with soundscape composition characteristics in similar type of city parks. These parameters include PLS of human sounds with $\mathrm{L}_{\mathrm{A} 10}$ $(+), \mathrm{L}_{\mathrm{Ceq}}-\mathrm{L}_{\text {Aeq }}(-)$ and sharpness $(+)$, respectively, POS of traffic sounds with $\mathrm{L}_{\mathrm{A} 10}(-)$, and PLS of both mechanical and geophysical sounds with sharpness $(+)$, with major positive or negative relationship marked in the bracket.

6. The relationships among these soundscape parameters also suggest the way to apply the soundscape composition parameters. By manipulating sound compositions, soundscapes with certain physical and psychoacoustic characteristics could be designed.

\section{Acknowledgements}

The authors would like to thank all the participants who were involved in the field work. Yiying Hao, Ming Yang and Joe Kang are acknowledged for useful discussions. Special thanks to Dr. Tao Luo for his support to the investigation. The work was supported by the State Science and Technology Programme (2013BAJ12B02).

\section{References}

Chiesura, A. 2004. The role of urban parks for the sustainable city, Landscape and Urban Planning 68(1): 129-138. http://dx.doi.org/10.1016/j.landurbplan.2003.08.003

De Coensel, B.; Botteldooren, D. 2006. The quiet rural soundscape and how to characterize it, Acta Acustica United with Acustica 92(6): 887-897.

De Coensel, B.; Vanwetswinkel, S.; Botteldooren, D. 2011. Effects of natural sounds on the perception of road traffic noise, The Journal of the Acoustical Society of America 129(4): 148-153. http://dx.doi.org/10.1121/1.3567073

Dubois, D.; Guastavino, C.; Raimbault, M. 2006. A cognitive approach to urban soundscapes: using verbal data to access everyday life auditory categories, Acta Acustica United with Acustica 92(6): 865-874.

Fowler, M. D. 2013. Soundscape as a design strategy for landscape architectural praxis, Design Studies 34(1): 111-128. http://dx.doi.org/10.1016/j.destud.2012.06.001 
Genuit, K.; Fiebig, A. 2006. Psychoacoustics and its benefit for the soundscape approach, Acta Acustica United with Acustica 92(6): 952-958.

Hall, D. A.; Irwin, A.; Edmondson-Jones, M.; Phillips, S.; Poxon, J. E. W. 2013. An exploratory evaluation of perceptual, psychoacoustic and acoustical properties of urban soundscapes, Applied Acoustics 74(2): 248-254. http://dx.doi.org/10.1016/j.apacoust.2011.03.006

Jeon, J. Y.; Lee, P. J.; You, J.; Kang, J. 2010. Perceptual assessment of quality of urban soundscapes with combined noise sources and water sounds, The Journal of the Acoustical Society of America 127: 1357-1366. http://dx.doi.org/10.1121/1.3298437

Kang, J.; Zhang, M. 2010. Semantic differential analysis of the soundscape in urban open public spaces, Building and Environment 45(1): 150-157. http://dx.doi.org/10.1016/j.buildenv.2009.05.014

Lavandier, C.; Defréville, B. 2006. The contribution of sound source characteristics in the assessment of urban soundscapes, Acta Acustica United with Acustica 92(6): 912-921.

Leventhall, H. 2004. Low frequency noise and annoyance, Noise and Health 6(23): 59-72.

Liu, J.; Kang, J.; Behm, H.; Luo, T. 2014. Effects of landscape on soundscape perception: Soundwalks in city parks, Landscape and Urban Planning 123: 30-40. http://dx.doi.org/10.1016/j.landurbplan.2013.12.003

Liu, J.; Kang, J.; Luo, T.; Behm, H. 2013a. Landscape effects on soundscape experience in city parks, Science of the Total Environment 454: 474-481. http://dx.doi.org/10.1016/j.scitotenv.2013.03.038

Liu, J.; Kang, J.; Luo, T.; Behm, H.; Coppack, T. 2013b. Spatiotemporal variability of soundscapes in a multiple functional urban area, Landscape and Urban Planning 115: 1-9. http://dx.doi.org/10.1016/j.landurbplan.2013.03.008
Matsinos, Y.; Mazaris, A.; Papadimitriou, K.; Mniestris, A.; Hatzigiannidis, G.; Maioglou, D.; Pantis, J. 2008. Spatio-temporal variability in human and natural sounds in a rural landscape, Landscape Ecology 23(8): 945-959. http://dx.doi.org/10.1007/s10980-008-9250-7

Raimbault, M. 2006. Qualitative judgements of urban soundscapes: questionning questionnaires and semantic scales, Acta Acustica United with Acustica 92(6): 929-937.

Raimbault, M.; Dubois, D. 2005. Urban soundscapes: experiences and knowledge, Cities 22(5): 339-350. http://dx.doi.org/10.1016/j.cities.2005.05.003

Rychtáriková, M.; Vermeir, G. 2013. Soundscape categorization on the basis of objective acoustical parameters, Applied Acoustics 74(2): 240-247. http://dx.doi.org/10.1016/j.apacoust.2011.01.004

Schafer, R. M. 1969. The new soundscape: a handbook for the modern music teacher. BMI Canada Don Mills, Ont.

Schulte-Fortkamp, B. 2002. The meaning of annoyance in relation to the quality of acoustic environments, Noise and Health 4(15): 13-18.

Thompson, C. W. 2002. Urban open space in the 21st century, Landscape and Urban Planning 60(2): 59-72. http://dx.doi.org/10.1016/S0169-2046(02)00059-2

Yang, W.; Kang, J. 2005a. Acoustic comfort evaluation in urban open public spaces, Applied Acoustics 66(2): 211-229. http://dx.doi.org/10.1016/j.apacoust.2004.07.011

Yang, W.; Kang, J. 2005b. Soundscape and sound preferences in urban squares: a case study in Sheffield, Journal of Urban Design 10(1): 61-80. http://dx.doi.org/10.1080/13574800500062395

Yu, L.; Kang, J. 2008. Effects of social, demographical and behavioral factors on the sound level evaluation in urban open spaces, The Journal of the Acoustical Society of America 123: 772-783. http://dx.doi.org/10.1121/1.2821955

Jiang LIU is an associated professor at the Fuzhou University, School of Architecture. He finished his PhD at the faculty of Agricultural and Environmental Sciences, University of Rostock, Germany, and did a guest research at the School of Architecture, University of Sheffield. His research interest is on soundscapes in relation to landscape planning and design. He has published 8 refereed journal papers.

Jian KANG has been Professor of Acoustics at the University of Sheffield, School of Architecture since 2003 and also holds a Qianren Professorship at the Harbin Institute of Technology. He obtained his $\mathrm{PhD}$ from the University of Cambridge. He has published 3 books, over 200 refereed journal papers and book chapters, and over 600 conference papers and technical reports. 